\title{
Morphological Analysis of the Rainbow Patterns Created by Point Defects of Graphene
}

\author{
M. Haždijojić1,* , M Ćosić1 ${ }^{1}$ and R. Rymzhanov ${ }^{2,3}$ \\ ${ }^{1}$ Laboratory of Physics, Vinča Institute of Nuclear Sciences, the University of Belgrade, \\ P. O. Box 522, 11001 Belgrade, Serbia \\ ${ }^{2}$ Joint Institute for Nuclear Research, Joliot-Curie 6, 141980 Dubna, Moscow Region, \\ Russia \\ ${ }^{3}$ The Institute of Nuclear Physics, Ibragimov St. 1, 050032 Almaty, Kazakhstan

\section{Supporting Information}

\section{Appendix A: Fluctuations of the scattering angle}

In the spherical coordinate system, the direction of the incoming proton is specified by polar angle $\Theta$, and azimuth $\Phi$. We assume that the distribution of deviations of the incoming protons from the $z$ axis is axially symmetric of small angular divergence. In that case, the distribution of incoming angles is given by a product of Rayleigh distribution

$$
r_{\sigma}(\Theta)=\frac{\Theta}{\sigma_{\theta}^{2}} \exp \left[-\frac{\Theta^{2}}{2 \sigma_{\theta}^{2}}\right],
$$

of small parameter $\sigma_{\theta}$, and a uniform distribution $u(\Phi)=1 / 2 \pi$.

In the laboratory coordinate systems, the scattering angle vector $\boldsymbol{\theta}$ is now a function of the impact parameter vector $\boldsymbol{b}$, and polar and azimuth angles $\Theta$, and $\Phi$. To evaluate the scattering law it is necessary to rotate the laboratory system by angle $\Theta$ around its $y$-axis by the matrix

$$
\boldsymbol{T}_{\Theta}=\left(\begin{array}{ccc}
\cos \Theta & 0 & \sin \Theta \\
0 & 1 & 0 \\
-\sin \Theta & 0 & \cos \Theta
\end{array}\right),
$$

followed by a rotation by an angle $\Phi$ around its $z$-axis given by the matrix

\footnotetext{
* Corresponding author. E-mail: milivoje@vin.bg.ac.rs
} 


$$
\boldsymbol{T}_{\Phi}=\left(\begin{array}{ccc}
\cos \Phi & -\sin \Phi & 0 \\
\sin \Phi & \cos \Phi & 0 \\
0 & 0 & 1
\end{array}\right)
$$

Obtained $z^{\prime}$-axis of the rotated coordinate system, coincides with the direction of the incoming proton. In the rotated coordinate system scattering angles $\boldsymbol{\theta}^{\prime}$ are obtained by evaluation of Eq. (5), using appropriate positions of atoms $\boldsymbol{R}_{n}^{\prime}$ and impact parameter $\boldsymbol{b}^{\prime}$ obtained by projection of rotated vectors $\boldsymbol{T}_{\Phi} \boldsymbol{T}_{\Theta} \boldsymbol{R}_{\boldsymbol{n}}$ and $\boldsymbol{T}_{\Phi} \boldsymbol{T}_{\Theta} \boldsymbol{b}$ on the $x^{\prime} 0 y^{\prime}$ plane by matrix

$$
\boldsymbol{P}=\left(\begin{array}{lll}
1 & 0 & 0 \\
0 & 1 & 0 \\
0 & 0 & 0
\end{array}\right)
$$

The scattering angles in the laboratory coordinate system $\boldsymbol{\theta}(\boldsymbol{b} ; \Theta, \Phi)$ are given by relation $\boldsymbol{\theta}$ $(\boldsymbol{b} ; \Theta, \Phi)=\boldsymbol{T}_{\Theta}^{T} \boldsymbol{T}_{\Phi}^{T} \boldsymbol{\theta}^{\prime}\left(\boldsymbol{b}^{\prime}\right)$.

The mean value of the scattering angle and its covariance matrix are given by the following integrals

$$
\overline{\boldsymbol{\theta}}(\boldsymbol{b})=\int \boldsymbol{\theta}(\boldsymbol{b}, \Theta, \Phi) r_{\sigma_{\theta}}(\Theta) u(\Phi) \Theta d \mathrm{~d} \Phi
$$

and

$$
\boldsymbol{\Sigma}(\boldsymbol{b})=\int[\boldsymbol{\theta}(\boldsymbol{b}, \Theta, \Phi)-\overline{\boldsymbol{\theta}}(\boldsymbol{b})] \cdot[\boldsymbol{\theta}(\boldsymbol{b}, \Theta, \Phi)-\overline{\boldsymbol{\theta}}(\boldsymbol{b})]^{T} r_{\sigma_{\theta}}(\Theta) u(\Phi) \Theta d \mathrm{~d} \Phi
$$

Direct evaluation of Eqs. (A1) and (A2) is impossible, however using the fact that $\sigma_{\theta}$ is small it is possible to derive simple analytical approximations.

Note that all values of $\Theta$ contributing significantly to integrals (A1) and (A2) are also small quantities. Next, note that for very small tilt angles $\Theta$ rotation by an angle $\Phi$ simply rotates rainbow pattern around the coordinate origin in the scattering angle plane $\theta_{x}^{\prime} 0 \theta_{y}{ }^{\prime}$ by the same angle. Thus, we introduce new auxiliary coordinate system whose $y^{\prime \prime}$-axis is parallel to the $y$-axis of the laboratory coordinate system. In this coordinate system, the scattering law given by Eq. (5) has the simplest mathematical form (since in it $\Phi=0$ ). Scattering angle vector in the rotated coordinate system $\boldsymbol{\theta}^{\prime}\left(\boldsymbol{b}^{\prime}\right)$ is given by relation 


$$
\boldsymbol{\theta}^{\prime}\left(\boldsymbol{b}^{\prime}\right)=\left(\begin{array}{cc}
\cos \Phi & \sin \Phi \\
-\sin \Phi & \cos \Phi
\end{array}\right) \boldsymbol{\theta}^{\prime \prime}\left(\boldsymbol{b}^{\prime \prime}\right)
$$

where $\boldsymbol{\theta}^{\prime \prime}$ is the scattering angle in the auxiliary coordinate system. Putting all thigh together we obtain the explicit expression for the scattering law which is too cumbersome to be written explicitly. Expanding obtained multivariate function $\boldsymbol{\theta}(\boldsymbol{b}, \Theta, \Phi)$ into a power series up to the second order one gets

$$
\boldsymbol{\theta}(\boldsymbol{b}, \Theta, \Phi)=\boldsymbol{a}_{0}(\Phi, b)+\boldsymbol{a}_{1}(\Phi, b) \Theta+\boldsymbol{a}_{2}(\Phi, b) \Theta^{2}+\sigma\left(\Theta^{3}\right) .
$$

where expansion coefficients $\boldsymbol{a}_{0}, \boldsymbol{a}_{1}$, and $\boldsymbol{a}_{2}$ are vector quantities. Substituting Eq. (A3) into Eq. (A1), and using the following trigonometric integrals [1]

$$
\int_{0}^{2 \pi} \sin ^{m} \Phi \cos ^{n} \Phi \mathrm{d} \Phi=\frac{n-1}{m+n} \int_{0}^{2 \pi} \sin ^{m} \Phi \cos ^{n-2} \Phi \mathrm{d} \Phi,
$$

and Gaussian integrals [1]

$$
\int_{0}^{\infty} r_{\sigma_{\theta}}(\Theta) \mathrm{d} \Theta=1, \int_{0}^{\infty} \Theta r_{\sigma_{\theta}}(\Theta) \mathrm{d} \Theta=\sqrt{\frac{\pi}{2}} \sigma_{\theta}, \int_{0}^{\infty} \Theta^{2} r_{\sigma_{\theta}}(\Theta) \mathrm{d} \Theta=2 \sigma_{\theta}^{2},
$$

the mean value of scattering angle becomes

$$
\overline{\boldsymbol{\theta}}(\boldsymbol{b})=\boldsymbol{\theta}_{\mathbf{0}}+\sigma_{\theta}^{2}\left[\frac{3}{2} \boldsymbol{\theta}_{\mathbf{0}}+2 \boldsymbol{\theta}_{\mathbf{1}}+\boldsymbol{\sigma}\left(\left|\boldsymbol{\theta}_{\mathbf{0}}\right|^{3}\right)\right],
$$

where $\boldsymbol{\theta}_{\mathbf{0}}=\boldsymbol{\theta}(\boldsymbol{b}, 0,0)$ the scattering angle for parallel beam, while vector $\boldsymbol{\theta}_{\mathbf{1}}$ is given by a very complicated expression that has $\left|\boldsymbol{\theta}_{\mathbf{1}}\right| \sim\left|\boldsymbol{\theta}_{\mathbf{0}}\right|$. Therefore, the second term the preceding equation is negligible in comparison to the first giving,

$$
\bar{\theta}(b) \approx \theta_{0} .
$$

To get the covariance matrix $\boldsymbol{\Sigma}$ of scattering angle fluctuations it is necessary to substitute Eqs. (A3) and (A4) into Eq. (A2) and to evaluate obtained integrals. The resulting expression can be evaluated explicitly yielding the following result

$$
\boldsymbol{\Sigma}(\boldsymbol{b})=\sigma_{\theta}^{2} \boldsymbol{I}+\sigma_{\theta}^{2}\left(2+\left|\boldsymbol{\theta}_{\mathbf{0}}\right|^{2}\right) \boldsymbol{\theta}_{\mathbf{0}} \cdot \boldsymbol{\theta}_{\mathbf{0}}^{\boldsymbol{T}} .
$$


Note that all terms proportional to components of the vector $\boldsymbol{\theta}_{\mathbf{1}}$ cancel each other out. For small scattering angles, nondiagonal correction is proportional to the small quantity of the fourth order and can be neglected giving

$$
\boldsymbol{\Sigma}(\boldsymbol{b}) \approx \sigma_{\theta}^{2} \boldsymbol{I}
$$

\section{Appendix B: The ghost of the degenerate critical pint}

Figure S1 shows the equipotential lines of the reduced potential $\varphi(\boldsymbol{b})$ for monovacancy defect in the vicinity of the two inflection saddles that are located at intersections of the negative $\boldsymbol{b}_{\boldsymbol{y}}$ axis and rainbow lines $t$ and $p_{2}$, respectively from Fig. 4(a). These inflection-saddle points are in Figure $\mathrm{S} 1$ labeled $i_{1}$, and $i_{2}$, respectively.

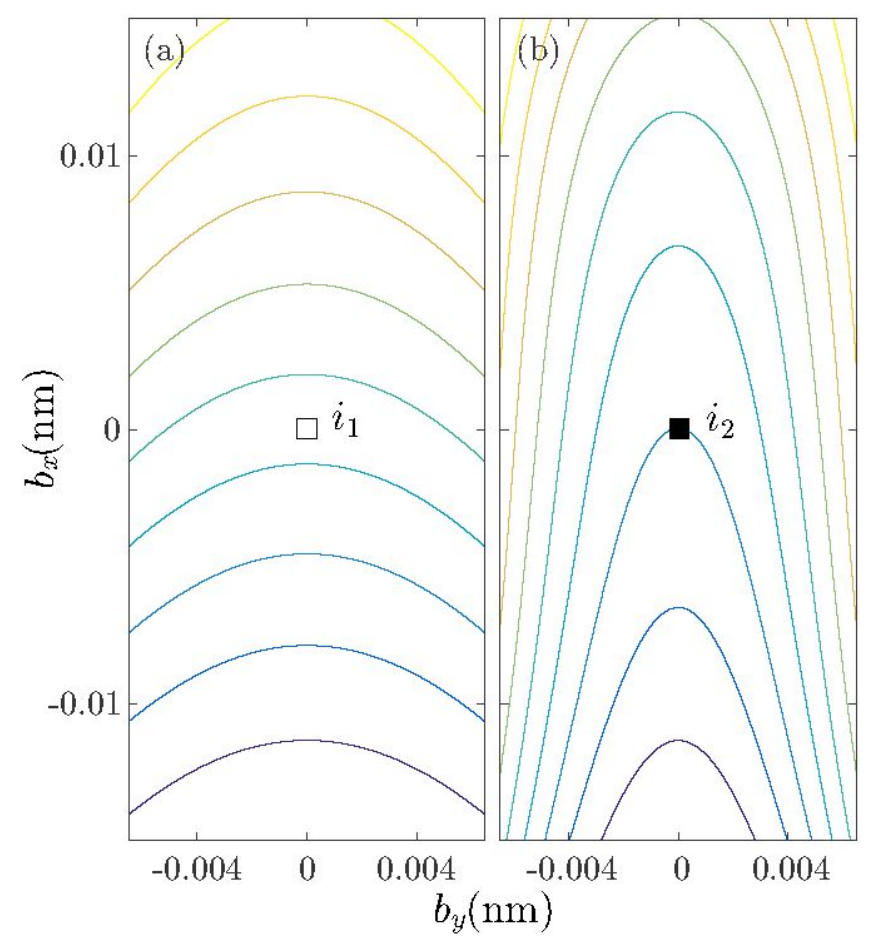

Figure S1 (color online) The potential lines of the reduced potential $\varphi(\boldsymbol{b})$ in the vicinity of the intersection of the negative $\boldsymbol{b}_{\boldsymbol{y}}$ axis and (a) rainbow line $t$, and (b) rainbow line $p_{2}$ from Fig. 4(a).

The appropriate model for the reduced potential, valid in the small vicinity of the inflection-saddle at $(0,0)$ is given by the fold catastrophe $A_{2}$ of the corank two given by the following bi-variate polynomial 


$$
\varphi(\boldsymbol{b}) \approx \alpha b_{y}+\frac{\beta}{3} b_{y}{ }^{3}+\frac{\gamma}{2} b_{x}{ }^{2}+\varphi_{0}
$$

where $\alpha, \beta$, and $\gamma$ are model parameters, and $\varphi_{0}=\varphi(0,0)$. Note that inflection saddle exists for $\alpha \cdot \beta>0, \gamma>0$. For $\alpha=0$, the inflection saddle transforms into the doubly degenerate critical point that for $\alpha \cdot \beta<0$, and $\gamma>0$ bifurcate into minimum-saddle pair.

The unknown values of the model parameters were obtained by the fitting procedure. For potential in the vicinity of the point $i_{1}$ optimal values of parameters are $\varphi_{0}=0.4965 \mathrm{eV}, \alpha=$ $3.0695 \mathrm{eV} / \mathrm{nm}, \beta=-1.5334 \times 10^{3} \mathrm{eV} / \mathrm{nm}^{3}$, and $\gamma=471.8732, \mathrm{eV} / \mathrm{nm}^{3}$, while optimal values of parameters for the inflection-saddle $i_{2}$ are $\varphi_{0}=0.6012 \mathrm{eV}, \alpha=0.3572 \mathrm{eV} / \mathrm{nm}, \beta=1.4188 \times$ $10^{3} \mathrm{eV} / \mathrm{nm}^{3}$, and $\gamma=566.0088, \mathrm{eV} / \mathrm{nm}^{3}$. Equipotential lines of obtained models are shown in Figure S2 (a), and Figure S2 (b) respectively. These Figs. show that a very simple model (B1) provides both qualitatively and quantitatively valid approximations of exact equipotential lines. Note that parameter $\alpha=0.3572 \mathrm{eV} / \mathrm{nm}$ of the model in the case of the point $i_{2}$ can be considered very small. Its equipotentials are practically indistinguishable from the equipotential lines in the degenerate critical case ( $\alpha=0)$ shown in Figure S2 (c). For the negative value of the parameter, of the slightly lower magnitude $\alpha=-0.0893$, (specifically chosen to provide equal density of equipotential lines) the topology of equipotential lines is radically different, as it is evident from Figure S2 (d).

In nonlinear dynamics, it was noticed that it takes an unusually long time for particles moving in the potential (B1) for $\alpha \cdot \beta>0, \gamma>0$, and $|\alpha|$ small to trasverse the region of the infection saddle. If particle dynamics is followed for the finite time, then particle trajectories can stay trapped in the examined region even there is no stationary point of the potential. Particle trajectories behave as if influenced by the ghost of the degenerate critical point. This is the reason why the inflection-saddle point $i_{2}$ is called the ghost critical point and labeled are labeled $g_{2}$ and $G_{2}$ in Fig. 4(a) respectively. 


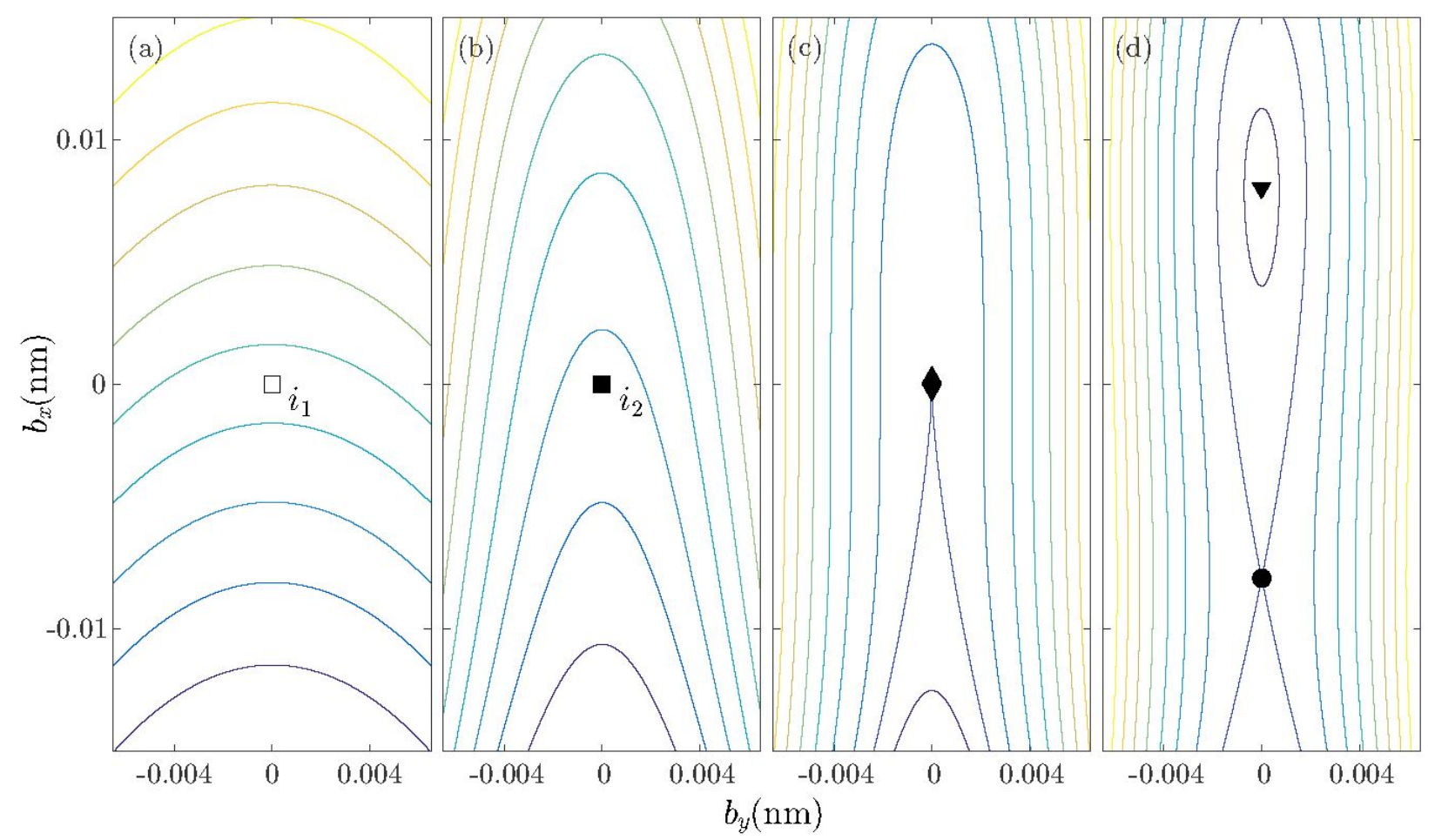

Figure S2 (color online) (a), (b) The equipotential of the catastrophic model of the potential in the vicinity of inflection-saddle points $i_{1}$ and $i_{2}$, respectively. (c) (d) Equipotential of the catastrophic model in the vicinity of the point $i_{2}$ for $\alpha=0$, and $\alpha=$ 0.0893 , respectively.

\section{References}

[1] M. Abramowitz and I. A. Stegun, Handbook of Mathematical Functions with Formulas, Graphs, and Mathematical Tables (National Bureau of Standards, Applied Mathematics Series $55,1964)$ 